\title{
Intestinal Perforation Caused by Lumboperitoneal Shunt Insertion Repaired with an Over-the-Scope Clip
}

\author{
Naoki Ishizuka and Eiji Komatsu \\ Department of Gastroenterology, Nishi-Yokohama International Hospital, Yokohama, Japan
}

Lumboperitoneal or ventriculoperitoneal shunt insertion is a standard therapy for hydrocephalus that diverts cerebrospinal fluid from the subarachnoid space into the peritoneal cavity. Gastrointestinal perforations due to this procedure occur rarely; however, accepted treatment strategies have not yet been established. Hence, the most common treatment approaches are open surgery or spontaneous closure without endoscopy. We report the case of a small intestinal perforation in a 73-year-old-woman that occurred after the insertion of a lumboperitoneal shunt. A positive cerebrospinal fluid culture and high cerebrospinal fluid white blood cell count indicated a retrograde bacterial infection, and computed tomography revealed that the peritoneal tip of the shunt catheter was located in the lumen of the gastrointestinal tract. We repaired the perforation endoscopically using an over-the-scope clip, and the patient's recovery was uneventful. Use of an over-the-scope clip could be an effective and minimally invasive treatment for intestinal perforations caused by lumboperitoneal or ventriculoperitoneal shunt insertion. Clin Endosc 2022;55:146-149

Key Words: Bacterial infection; Hydrocephalus; Intestinal perforation; Laparotomy; Ventriculoperitoneal shunt

\section{INTRODUCTION}

The standard treatment for hydrocephalus is lumboperitoneal (LP) or ventriculoperitoneal (VP) shunt insertion; the shunt diverts cerebrospinal fluid (CSF) from the subarachnoid space into the peritoneal cavity. However, the use of these shunts is associated with various complications, including infection and shunt obstruction. Intestinal perforations occur in $0.01 \%-0.07 \%$ of patients, mainly children, with VP shunts. ${ }^{1}$ Intestinal perforations can result in serious infections, sepsis, or even death. ${ }^{1,2}$ Therefore, they require prompt treatment. A laparotomy is advised for treating serious intra-abdominal infections. In the absence of peritonitis or an abdominal abscess,

Received: November 12, 2020 Revised: December 14, 2020

Accepted: December 18, 2020

Correspondence: Naoki Ishizuka

Current affiliation-Department of Internal Medicine, Hodogaya-Kita Clinic, 2-29-10 Nishiya, Hodogaya-ku, Yokohama, Kanagawa 240-0054, Japan

Tel: +81-45-382-0321, Fax: +81-45-382-0322, E-mail: n-ishizuka@houmonshinryou.com

ORCID: https://orcid.org/0000-0003-0978-9741

(c) This is an Open Access article distributed under the terms of the Creative Commons Attribution Non-Commercial License (http://creativecommons.org/ licenses/by-nc/3.0) which permits unrestricted non-commercial use, distribution, and reproduction in any medium, provided the original work is properly cited. the shunt catheter can be removed without surgery, ${ }^{1,3}$ although an abdominal abscess can occur after shunt removal. ${ }^{4}$ Recently, over-the-scope clips (OTSCs) were introduced for the non-surgical treatment of gastrointestinal perforations, fistulas, anastomotic leaks, and refractory gastrointestinal bleeding. Herein, we reported the case of a 73-year-old woman who had an LP shunt that perforated her gastrointestinal tract. The perforation was successfully closed using an OTSC.

\section{CASE REPORT}

A 73-year-old woman was admitted to our hospital with complaints of fever and headache that had persisted for a few days prior to admission. She had undergone an ascending colon/sigmoid colon anastomosis due to a large intestinal obstruction 3 years earlier; an LP shunt had been inserted due to idiopathic normal pressure hydrocephalus 1 year prior to presentation. About 3 months before the onset of her symptoms, she underwent revision of the LP shunt.

Physical examination revealed no inflammation of the skin overlying the abdominal course of the shunt catheter and no tenderness on palpation of the abdomen. Computed 
tomography (CT) of the head, performed on admission, did not indicate ventriculomegaly. Abdominal/pelvic CT revealed that the shunt catheter had been inserted into the abdominal cavity through the left lower quadrant (Fig. 1A) but had perforated the large intestine (Fig. 1B-D). There were no signs of intra-abdominal fluid collection, abscess formation, or free air within the peritoneum.

Analysis of LP shunt fluid revealed an ongoing bacterial in- fection. The fluid culture showed the presence of Enterobacter cloacae and Lactobacillus spp.; the patient had an increased white blood cell count $(2,021 / \mu \mathrm{L})$, indicating CSF infection. Administration of meropenem hydrate (6 g/day) was started to contain the infection. The catheter was cut at the point where the LP shunt was inserted into the abdominal wall, and the cranial section of the shunt was removed. We warned the patient and her family of the possibility of the leakage of intes-
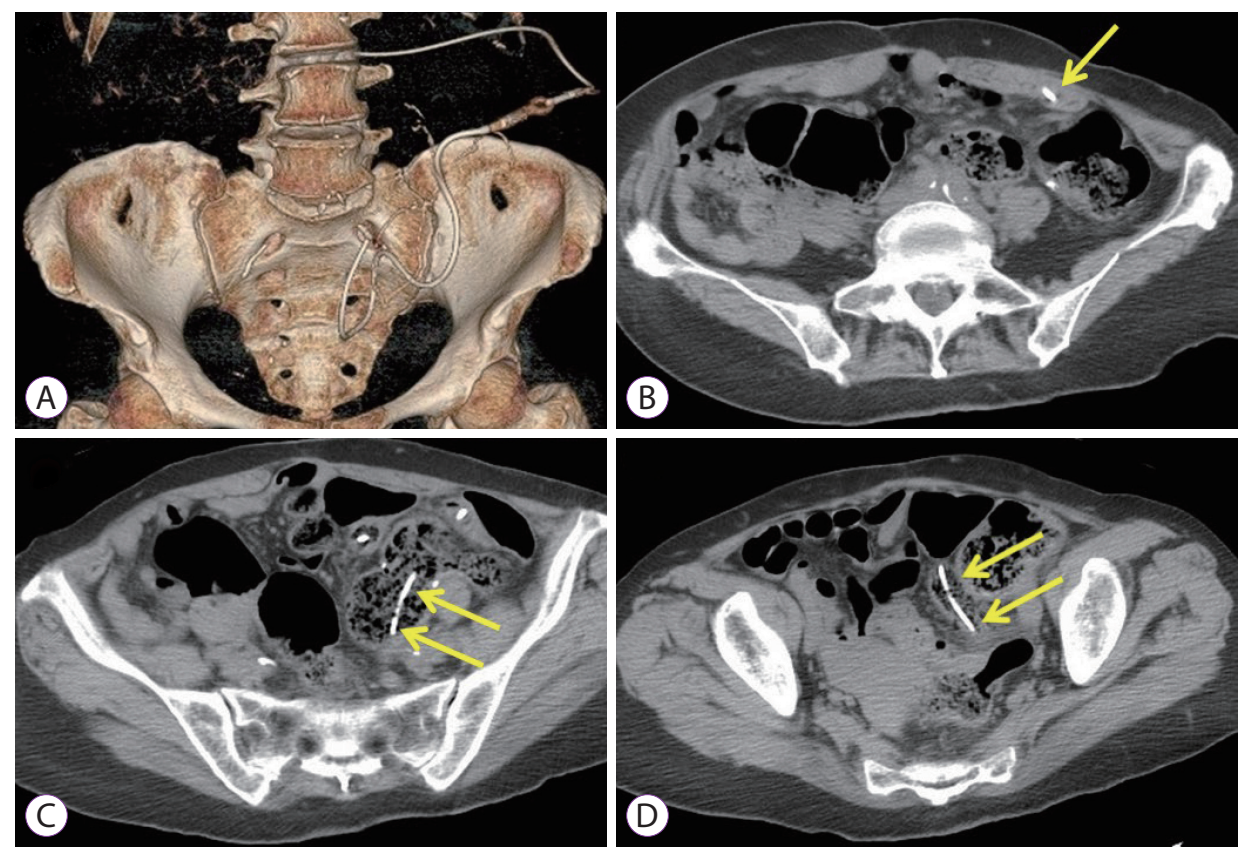

Fig. 1. Abdominal/pelvic computed tomography on admission showing the shunt catheter penetrating the abdominal wall and its location in the gastrointestinal tract. (A) Entry of the lumboperitoneal shunt catheter into the abdominal cavity from the lower left abdomen. (B-D) Location of the shunt catheter in the intestinal tract (arrows indicate the shunt catheter).
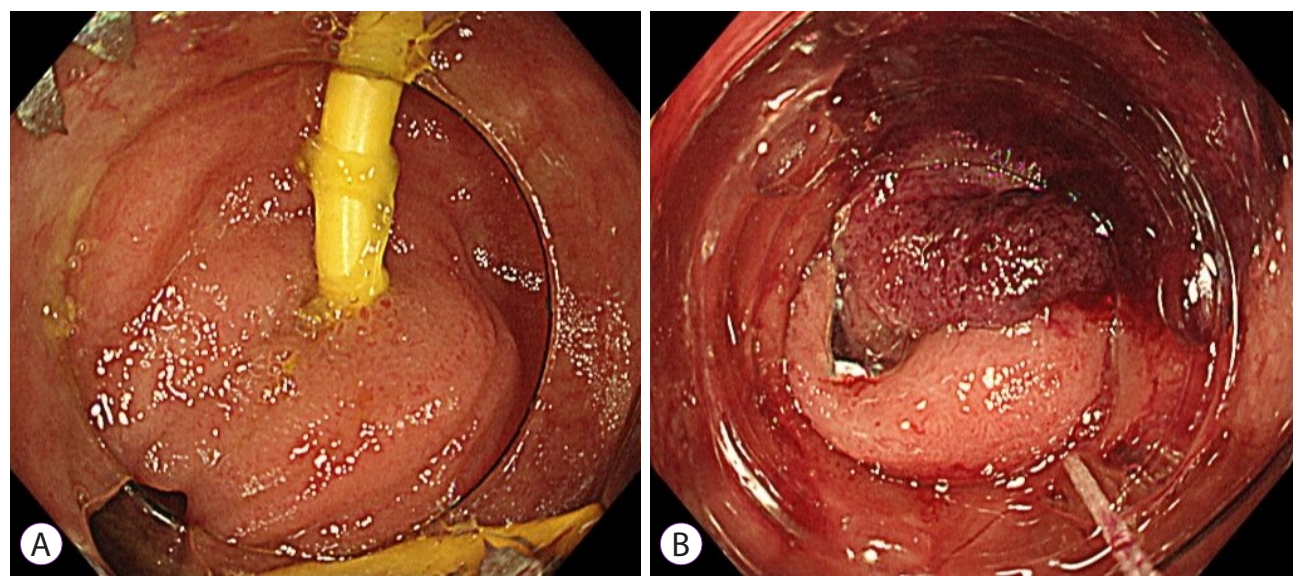

Fig. 2. Endoscopic images taken before and after repairing the perforation site with an over-the-scope-clip (OTSC). (A) The shunt catheter is penetrating the ileal wall and is located within the intestinal tract. (B) The perforation site is closed using an OTSC. 

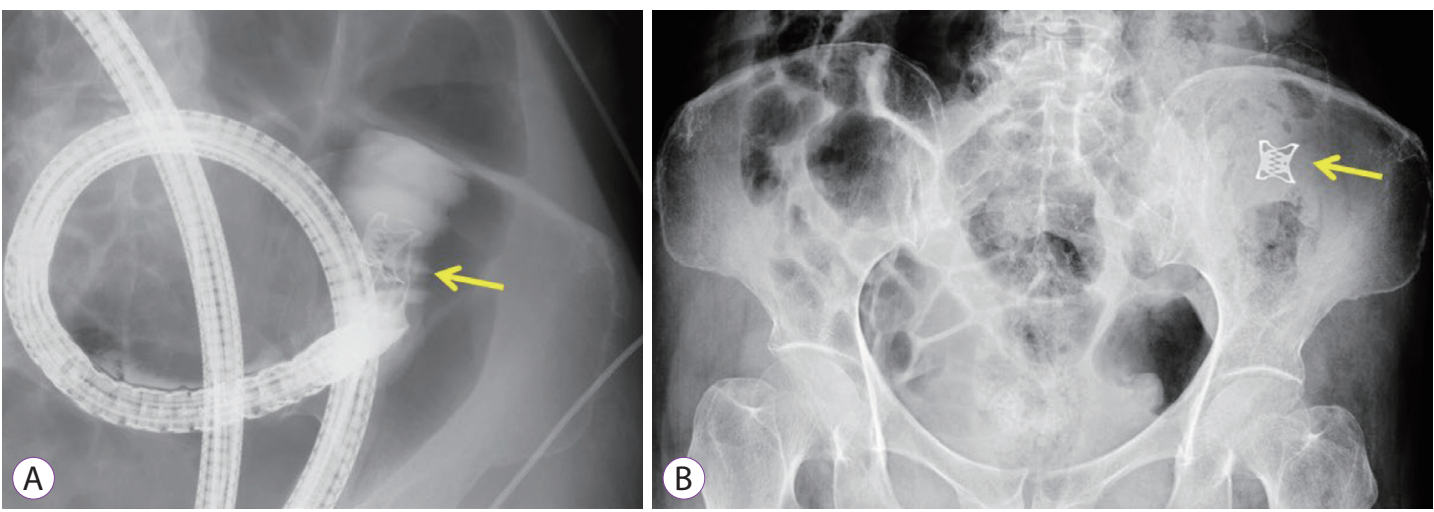

Fig. 3. Abdominal radiographs acquired after the use of an over-the-scope-clip (OTSC) (arrows indicate the OTSC). (A) Confirming the closure of the perforation by administering a gastrografin infusion through the endoscope. (B) Plain abdominal radiography showing successful OTSC use.

tinal contents upon removal of the remaining section, which could lead to peritonitis requiring laparotomy. We explained that peritonitis could be avoided by suturing the perforated part of the intestine when the catheter was removed and that the use of an OTSC was a less invasive, reliable, and affordable (covered by insurance) treatment option for gastrointestinal perforation. All procedures were performed by a senior endoscopist in the department of gastroenterology at our hospital. Informed consent was obtained from the patient after providing a full explanation about the procedure and its complications.

Colonoscopy was performed with an OTSC system (Ovesco Endoscopy AG, Tübingen, Germany). Using an endoscope (CF TYPE H260I; Olympus, Tokyo, Japan), an OTSC (11 $\mathrm{mm} /$ type-t) was inserted into the cecum. The end of the shunt catheter was observed in the ileum; however, it could not be reached, as the diameter of the endoscope was wider than the patient's ileum. Therefore, a narrower endoscope (GIF TYPE Q260; Olympus) and OTSC (10 mm/type-t) were inserted. The shunt catheter was removed from its entry point in the ileum, $5 \mathrm{~cm}$ from the ileocecal valve (Fig. 2A), and an OTSC was used to close the gastrointestinal perforation (Fig. 2B). After the procedure, the absence of leakage was confirmed via the administration of a gastrografin infusion through the endoscope (Fig. 3A), and the correct placement of the OTSC was ascertained via plain radiography (Fig. 3B).

The patient was permitted to eat 5 days after the procedure. No adverse symptoms, such as abdominal pain and vomiting, due to food intake were recorded. A fever due to bacterial meningitis was noted, and antibiotics were administered (meropenem hydrate, $6 \mathrm{~g} /$ day until postoperative day 4 , and ceftriaxone sodium hydrate, $2 \mathrm{~g} /$ day from postoperative day 5 to 18). No movement of the OTSC clip was observed on plain abdominal radiography on postoperative day 19 , and the patient was discharged on postoperative day 20.

\section{DISCUSSION}

The insertion of LP and VP shunts is associated with various complications, such as meningitis, sepsis, pseudocyst formation, and intestinal perforations. Intestinal perforations, which chiefly occur in children, have an overall mortality rate of approximately $15 \%-18 \% .{ }^{1}$ The management of a case similar to the one described above depends on several factors, such as the presence of intra-abdominal abscesses, peritonitis, or meningitis. The treatment strategies for intestinal perforations caused by peritoneal shunt catheters include shunt removal, external drainage, and intravenous antibiotic administration. ${ }^{2}$ However, when intra-abdominal abscesses and peritonitis are present, a laparotomy or laparoscopic surgery should be performed. ${ }^{5}$ Surgical treatment is not required for asymptomatic patients with simple intestinal perforations; rather, the shunt catheter should be disconnected at the abdominal wall, and the distal end should be removed endoscopically. However, spontaneous closure of the perforated intestine is unpredictable because sufficient fibrosis may not occur around the catheter; therefore, a laparotomy should be performed when perforations do not close spontaneously after the percutaneous or endoscopic removal of the catheter. When the perforation is located near the anus or in the large intestine, endoscopic closure can help avoid the risks of surgery. Chiang et al. reported a case involving suturing of a perforation through the anus. ${ }^{6}$

In the present case, bacterial meningitis occurred due to 
an intestinal perforation caused by the catheter; however, there was no intraperitoneal abscess or peritonitis. Therefore, endoscopic treatment was indicated as the OTSC system ensures perforation closure and is effective in the treatment of gastrointestinal perforations, bleeding, and fistulas. Moreover, it has been reported that the use of OTSCs has reduced the rate of open surgical treatment for iatrogenic gastrointestinal perforations. ${ }^{7}$ Open surgery and its associated risks can be avoided, even when intra-abdominal abscesses or free air exist, by endoscopically closing the perforation using an OTSC and performing intraperitoneal drainage.

The clinical success rate of using OTSCs for gastrointestinal perforations is $84.6 \%$, while the complication rate is $1.7 \%$, with severe complications occurring in only $0.59 \%$ of patients. ${ }^{8}$ Inserting an OTSC in the small intestine is technically difficult; however, it has been reported to be effective in treating iatrogenic perforations of the duodenum and jejunum., ${ }^{9,10}$ OTSCs should be used cautiously for perforations in the small intestine, as small intestinal stenosis may occur after OTSC insertion.

In the present case, the perforation site was located at the distal end of the ileum, which was relatively easy to reach with an endoscope. After the catheter was removed, visualization of the perforation site in the ileum was difficult because of intestinal peristalsis, but perforations of up to $10 \mathrm{~mm}$ in diameter can be closed instantly with an OTSC, making this technique more reliable than the use of conventional clips.

To the best of our knowledge, this is the first report describing the use of an OTSC to treat an intestinal perforation caused by a shunt catheter. Use of an OTSC is an effective and minimally invasive management option for gastrointestinal perforations due to LP and VP shunts.

Conflicts of Interest

The authors have no potential conflicts of interest.

Funding

None.
Acknowledgments

We gratefully acknowledge the technical advice of Mr. Takuya Osu, Century Medical, Inc.

\section{Author Contributions}

Supervision: Eiji Komatsu

Writing-original draft: Naoki Ishizuka

Writing-review\&editing: NI

ORCID

Naoki Ishizuka

https://orcid.org/0000-0003-0978-9741

Eiji Komatsu

https://orcid.org/0000-0001-9804-3196

\section{REFERENCES}

1. Sathyanarayana S, Wylen EL, Baskaya MK, Nanda A. Spontaneous bowel perforation after ventriculoperitoneal shunt surgery: case report and a review of 45 cases. Surg Neurol 2000;54:388-396.

2. Vinchon M, Baroncini M, Laurent T, Patrick D. Bowel perforation caused by peritoneal shunt catheters: diagnosis and treatment. Neurosurgery 2006;58:S76-S82.

3. Martinez Hernández-Magro P, Barrera Román C, Villanueva Sáenz E, Zavala MJ. Colonic perforation as a complication of ventriculoperitoneal shunt: a case report. Tech Coloproctol 2006;10:353-355.

4. Sells CJ, Loeser JD. Peritonitis following perforation of the bowel: a rare complication of a ventriculoperitoneal shunt. J Pediatr 1973;83:823-824.

5. Knuth J, Detzner M, Heiss MM, Weber F, Bulian DR. Laparoscopy for a ventriculoperitoneal shunt tube dislocated into the colon. JSLS 2013;17:675-678

6. Chiang LL, Kuo MF, Fan PC, Hsu WM. Transanal repair of colonic perforation due to ventriculoperitoneal shunt--case report and review of the literature. J Formos Med Assoc 2010;109:472-475.

7. Khater S, Rahmi G, Perrod G, et al. Over-the-scope clip (OTSC) reduces surgery rate in the management of iatrogenic gastrointestinal perforations. Endosc Int Open 2017;5:E389-E394.

8. Kobara H, Mori H, Nishiyama N, et al. Over-the-scope clip system: a review of 1517 cases over 9 years. J Gastroenterol Hepatol 2019;34:22-30.

9. Voermans RP, Le Moine O, von Renteln D, et al. Efficacy of endoscopic closure of acute perforations of the gastrointestinal tract. Clin Gastroenterol Hepatol 2012;10:603-608.

10. Purchiaroni F, Nakajima T, Sakamoto T, Abe S, Saito Y. Over-thescope-clip pre-mounted onto a double balloon enteroscope for fast and successful closure of post-EMR jejunal perforation: case report. BMC Gastroenterol 2017;17:152. 